J. Peetre

Nagoya Math. J.

Vol. 130 (1993), 183-192

\title{
HANKEL KERNELS OF HIGHER WEIGHT FOR THE BALL
}

\author{
JAAK PEETRE
}

The purpose of this note is to write down the general form of Hankel kernels for the complex unit ball $\mathbf{B}$ in $\mathbf{C}^{d}$. In the one dimensional case (unit disk $\Delta$ in $\mathbf{C}$ ) this was done in [JP] and our treatment below has been guided by the insights gained there, and later, in a slightly different context, in [P]. We begin by summarizing the relevant facts in the case of the disk in a form convenient for us.

\section{The disk revisited}

Let $A^{\alpha, 2}$ be the Hilbert space of analytic functions on $\Delta$ which are square integrable with respect to the probability measure $d M_{a}(z)=(\alpha+1)$ $\left(1-|z|^{2}\right)^{\alpha} d \mathscr{E}(z)(\mathscr{E}=$ Euclidean measure with an appropriate normalization, viz. $\left.\int_{\Delta} d \mathscr{E}(z)=1\right)$. In $[\mathrm{JP}]$ we considered bilinear forms on $A^{\alpha, 2}(\Delta)$ of the form

$$
H\left(f_{1}, f_{2}\right)=\iint_{\Delta \times \Delta} \overline{\mathscr{A}\left(z_{1}, z_{2}\right)} f_{1}\left(z_{1}\right) f_{2}\left(z_{2}\right) d M_{a}\left(z_{1}\right) d M_{\alpha}\left(z_{2}\right),
$$

where the kernel $\mathscr{A}$ is given by

$$
\mathscr{A}\left(z_{1}, z_{2}\right)=\frac{1}{2 \pi i} \int_{\mathrm{T}} \frac{\left(z_{1}-z_{2}\right)^{s}}{\left(w-z_{1}\right)^{r}\left(w-z_{2}\right)^{r}} b(w) d w,
$$

where $r=\alpha+2+s(s \in \mathbf{N})$. We write now this formula as

$$
\mathscr{A}\left(z_{1}, z_{2}\right)=\frac{1}{2 \pi i} \int_{\mathrm{T}} \frac{\left(z_{1}-z_{2}\right)^{s}}{\left(1-\bar{w} z_{1}\right)^{r}\left(1-\bar{w} z_{2}\right)^{r}} b(w) \bar{w}^{2 r} d w .
$$

By partial integration one finds, quite generally, that

$$
\int_{\Delta} \bar{f} g d M_{m-1}=\frac{(m+1) !}{2 \pi i} \int_{\mathrm{T}} \bar{f} b \bar{w}^{m+1} d w
$$

Received June 13. 1988. 
where $g=b^{(m)}$. (Alternative proof: Just check it for the monomials. The (limiting) case $m=0$ is identity, while $m=1$ is essentially Green's formula.) If $m=2 r-1$ this gives

$$
\mathscr{A}\left(z_{1}, z_{2}\right)=\frac{1}{(2 r) !} \int_{\Delta} \frac{\left(z_{1}-z_{2}\right)^{s}\left(1-|w|^{2}\right)^{s}}{\left(1-z_{1} \bar{w}\right)^{r}\left(1-z_{2} \bar{w}\right)^{r}} g(w) d M_{\beta}(w)
$$

where $\beta=2(\alpha+2)+s-2$. (Notice the splitting of the $\left(1-|w|^{2}\right)$-factor, which is convenient for our purposes.) It is often convenient to set $\nu=\alpha+2$, $\mu=\beta+2$. Then the last relation reads $\mu=2 \nu+s$.

Remark. By partial integration

$$
\begin{gathered}
0=\int_{\Delta} \frac{d}{d z}\left(f \bar{g}\left(1-|z|^{2}\right)^{\alpha+1}\right) d \mathscr{E}(z) \\
=\int_{\Delta} f^{\prime} \bar{g}\left(1-|z|^{2}\right)^{\alpha+1} d \mathscr{E}(z)-\int_{\Delta} \frac{(\alpha+1) z f}{1-|z|^{2}} \bar{g}\left(1-|z|^{2}\right)^{\alpha+1} d \mathscr{E}(z)
\end{gathered}
$$

so that $f \mapsto f^{\prime}$ and $f \mapsto P_{a+1}\left(\frac{(\alpha+1) z f}{1-|z|^{2}}\right)$ are adjoint operators in $A^{\alpha+1}(\Delta), P_{a+1}$ being the corresponding orthogonal projection. Therefore, if $s=1$, the bilinear form can be written in the form

$$
H\left(f_{1}, f_{2}\right)=\text { const } \cdot \int_{\Delta}\left(f_{1}^{\prime} f_{2}-f_{1} f_{2}^{\prime}\right) \bar{g}\left(1-|z|^{2}\right)^{s} d M_{\beta}(z)
$$

In a similar way we recover, if $s>1$, quite generally the transvectant (German: Überschiebung). This replaces, in one stroke, the rather clumsy attempts in [JP], Section 3.4.

\section{The ball}

Let $\mathbf{B}$ denote the unit ball in $\mathbf{C}^{d}$. The measure $\mathcal{M}_{\alpha}(\alpha>-1)$ and the Hilbert space $A^{\alpha, 2}(\mathbf{B})$ are defined in an analogous way as for the disk (Section 1).

Let us begin with the case $s=1$.

"ANSATZ".

$$
\mathscr{A}\left(z_{1}, z_{2}\right)=\mathscr{A}_{g}\left(z_{1}, z_{2}\right)=\int_{\mathbf{B}} \sum_{i=1}^{d} A^{i}\left(z_{1}, z_{2}, w\right) g_{i}(w) d \mathcal{M}_{\beta}(w)
$$

where $\beta=2(\alpha+1+d)+1-(d+1)$. It will be convenient to put $\nu=\alpha+d+1$, 
$\mu=\beta+d+1$. Then the preceding relation reads $\mu=2 \nu+1$. (This agrees with the convention made (for general $s$ ) in Section 1 if $d=1$.)

Let

$$
\left(\begin{array}{c}
d \times(d+1) \\
D \\
1 \times(d+1) \\
E
\end{array}\right) \in \mathrm{SU}(d, 1)
$$

writing $\phi z=\frac{D(z)}{E(z)}$ with $D(z)=D\left(\begin{array}{l}z \\ 1\end{array}\right), E(z)=E\left(\begin{array}{l}z \\ 1\end{array}\right)$. Then

$$
\begin{gathered}
f(z) \mapsto f(\phi z)(E(z))^{-\nu} \equiv \tilde{f}(z), \\
\mathscr{A}\left(z_{1}, z_{2}\right) \mapsto \mathscr{A}\left(\phi z_{1}, \phi z_{2}\right)\left(E\left(z_{1}\right)\right)^{-\nu}\left(E\left(z_{2}\right)\right)^{-\nu} \equiv \tilde{\mathscr{A}}\left(z_{1}, z_{2}\right) .
\end{gathered}
$$

We want the $g_{i}$ to transform as components of a weighted differential form:

$$
g_{i}(w) \mapsto \sum_{j=1}^{d} g_{j}(\phi w) \frac{\partial \phi^{j}(w)}{\partial w^{2}}(E(w))^{-2 \nu} \equiv \bar{g}_{\imath}(w)
$$

This requires that

$$
\begin{aligned}
\mathscr{A}^{i}\left(\phi z_{1}, \phi z_{2}, \phi w\right)= & \sum_{j=1}^{d} \mathscr{A}^{j}\left(z_{1}, z_{2}, w\right) \frac{\partial \phi^{i}(w)}{\partial w^{j}} \times \\
& \times\left(E\left(z_{1}\right)\right)^{\nu}\left(E\left(z_{2}\right)\right)^{\nu}(\overline{E(w)})^{\mu} E(w) .
\end{aligned}
$$

Indeed, if (1) is fulfilled we find, using $d M_{\beta}(\phi w)=|E(w)|^{-2 \mu} d M_{\beta}(w)$,

$$
\begin{aligned}
\tilde{\mathscr{A}}\left(z_{1}, z_{2}\right)= & \int_{\mathbf{B}} \sum_{i=1}^{d} A^{i}\left(\phi z_{1}, \phi z_{2}\right) g_{i}(w) d M_{\beta}(\phi w) \cdot\left(E\left(z_{1}\right)\right)^{-\nu}\left(E\left(z_{2}\right)\right)^{-\nu} \\
= & \int_{\mathbf{B}} \sum_{i=1}^{d} \sum_{j=1}^{d} A^{j}\left(z_{1}, z_{2}, w\right) \frac{\partial \phi^{i}(w)}{\partial w^{j}}\left(E\left(z_{1}\right)\right)^{\nu}\left(E\left(z_{2}\right)\right)^{\nu}(\overline{E(w)})^{\mu} E(w) \\
& \quad \times g_{i}(w)|E(w)|^{-2 \mu} d M_{\beta}(w) \cdot\left(E\left(z_{1}\right)\right)^{-\nu}\left(E\left(z_{2}\right)\right)^{-\nu} \\
= & \int_{\mathbf{B}} \sum_{j=1}^{d} A^{j}\left(z_{1}, z_{2}, w\right) \tilde{g}_{j}(w) d M_{\beta}(w) .
\end{aligned}
$$

Example. If $d=1$ this is fulfilled with

$$
\mathscr{A}\left(z_{1}, z_{2}, w\right)=\frac{\left(z_{1}-z_{2}\right)\left(1-|w|^{2}\right)}{\left(1-z_{1} \bar{w}\right)^{\alpha+2+1}\left(1-z_{2} \bar{w}\right)^{\alpha+2+1}},
$$

in full agreement with Section 1. 
NEW "ANSATZ". $\quad \mathscr{A}^{i}\left(z_{1}, z_{2}, 0\right)=z_{1}^{i}-z_{2}^{i}$.

Clearly (1) is then fulfilled if $\phi$ fixes the origin 0 (a rotation). Next we apply (1) to the fundamental symmetry interchanging $w$ and 0 . For simplicity we first assume $w=(t, 0, \ldots, 0), t>0$. Then (see $[\mathrm{R}] ; i, j$ denote indices $>1$ )

$$
\begin{gathered}
\phi(z)=\left(\frac{t-z^{1}}{1-t z^{1}},-\left(1-t^{2}\right)^{1 / 2} \frac{z^{1}}{1-t z^{1}}\right), \\
\frac{\partial \phi}{\partial z^{1}}=\left(\frac{t^{2}-1}{\left(1-t z^{1}\right)^{2}}, \frac{\left(1-t^{2}\right)^{1 / 2} t z^{i}}{\left(1-t z^{1}\right)^{2}}\right), \\
\frac{\partial \phi}{\partial z^{j}}=\left(0,-\left(1-t^{2}\right)^{1 / 2} \frac{\delta_{j}^{l}}{1-t z^{1}}\right), \\
E(z)=\iota \frac{1-t \frac{t-z^{1}}{1-t z^{1}}}{\left(1-t^{2}\right)^{1 / 2}}=\iota \frac{\left(1-t^{2}\right)^{\frac{1}{2}}}{1-t z^{1}}, \\
E(0)=\frac{\iota}{\left(1-t^{2}\right)^{\frac{1}{2}}}, \quad
\end{gathered}
$$

where we in the last implication used $\phi^{2}=\mathrm{id}$ and the notation $\iota=(-1)^{\frac{d+1}{2}}$.

Writing $A=\left(A^{1}, \ldots, A^{d}\right)$ we find

$$
\begin{gathered}
A\left(z_{1}, z_{2}, w\right)=\left(A^{i}\left(z_{1}, z_{2}, w\right)\right) \\
=\left(\sum_{j=1}^{d} A^{j}\left(\phi^{-1} z_{1}, \phi^{-1} z_{2}, 0\right) \frac{\partial \phi^{2}(0)}{\partial w^{j}}\right) \times\left(E\left(\phi^{-1} z_{1}\right)\right)^{\nu}\left(E\left(\phi^{-1} z_{2}\right)\right)^{\nu}(\overline{E(0)})^{\mu} E(0) \\
=\left\{\left(\frac{t-z_{1}^{1}}{1-t z_{1}^{1}}-\frac{t-z_{2}^{1}}{1-t z_{2}^{1}}\right)\left(t^{2}-1,0\right)\right. \\
\left.+\sum_{j=2}^{d}\left(-\left(1-t^{2}\right)^{\frac{1}{2}} \frac{z_{1}^{j}}{1-t z_{1}^{1}}+\left(1-t^{2}\right)^{\frac{1}{2}} \frac{z_{2}^{j}}{1-t z_{2}^{1}}\right)\left(0,-\left(1-t^{2}\right)^{\frac{1}{2}} \delta_{i}^{j}\right)\right\} \\
\times \frac{\left(1-t^{2}\right)^{\nu}\left(1-t^{2}\right)^{-(\nu+1)}}{\left(1-t z_{1}^{1}\right)^{\nu}\left(1-t z_{2}^{1}\right)^{\nu}} .
\end{gathered}
$$

The two numerical factors in front of the vector terms can be written as

$$
\frac{\left(t^{2}-1\right)\left(z_{1}^{1}-z_{2}^{1}\right)}{\left(1-t z_{1}^{1}\right)\left(1-t z_{2}^{1}\right)} \text { and }-\left(1-t^{2}\right)^{\frac{1}{2}} \frac{z_{2}^{j}-z_{2}^{j}-t\left(z_{2}^{1} z_{1}^{j}-z_{1}^{1} z_{2}^{j}\right)}{\left(1-t z_{1}^{1}\right)\left(1-t z_{2}^{1}\right)}
$$

Therefore 


$$
\left\{\begin{array}{l}
A^{1}=\frac{\left(z_{1}^{1}-z_{2}^{2}\right)\left(1-t^{2}\right)}{\left(1-t z_{1}^{1}\right)^{\nu+1}\left(1-t z_{2}^{1}\right)^{\nu+1}} \\
A^{i}=\frac{z_{1}^{i}-z_{2}^{i}-t\left(z_{2}^{1} z_{1}^{i}-z_{1}^{1} z_{2}^{i}\right)}{\text { same denominator }} \quad(i>1) .
\end{array}\right.
$$

If $w$ is general, these formulae may be written as

$$
A=\frac{P_{w}\left(z_{1}-z_{2}\right)\left(1-|w|^{2}\right)+Q_{w}\left(z_{1}-z_{2}\right)-\left(\left(z_{2}, \bar{w}\right) z_{1}-(z, \bar{w}) z_{2}\right)}{\left(1-\left(z_{1}, \bar{w}\right)\right)^{\nu+1}\left(1-\left(z_{2}, \bar{w}\right)\right)^{\nu+1}}
$$

where $P_{w}$ is orthogonal projection in the $w$ direction and $Q_{w}=1-P_{w}$;

$$
|w|^{2}=\sum_{j=1}^{d}\left|w^{j}\right|^{2}, \quad(z, \bar{w})=\sum_{j=1}^{d} z^{j} \bar{w}^{j}
$$

is the standard Hermitean metric in $\mathbf{C}^{d}$. Explicitly:

$$
P_{w}(\cdot)=\left(\cdot, \frac{w}{|w|}\right) \frac{w}{|w|}
$$

so that we can as well write

$$
A=\frac{\left(1-\left(z_{2}, \bar{w}\right)\right) z_{1}-\left(1-\left(z_{1}, \bar{w}\right)\right) z_{2}-\left(z_{1}-z_{2}, \bar{w}\right) w}{\text { previous denominator }} .
$$

We can now make the Janson remark (see $[\mathrm{P}]$ ) and pass to homogeneous notation. Writing $A=\left(A^{1}, \ldots, A^{d}\right), g=\left(g_{1}, \ldots, g_{d}\right),\langle A, g\rangle=\sum_{i=1}^{d} A^{i} g_{i}$ and putting

$$
g_{0}=-\sum_{i=1}^{d} g_{i} w^{i}
$$

we get

$$
\langle A, g\rangle=\frac{g_{0}\left(z_{1}-z_{2}, \bar{w}\right)+\left\langle g,\left(1-\left(z_{2}, \bar{w}\right)\right) z_{1}-\left(1-\left(z_{1}, \bar{w}\right)\right) z_{2}\right\rangle}{\text { previous denominator }} .
$$

Write further

$$
Z_{k}=\left(1, z_{k}^{1}, \ldots, z_{k}^{d}\right)(k=1,2), \quad W=\left(1, w^{1}, \ldots, w^{d}\right), \quad G=\left(g_{0}, \ldots, g_{d}\right)
$$

and consider the standard pseudo-Hermitean metric in $\mathbf{C}^{d+1}$ :

$$
\begin{gathered}
\|Z\|^{2}=\left|Z^{0}\right|^{2}-\left|Z^{1}\right|^{2}-\cdots-\left|Z^{d}\right|^{2}, \\
((Z, \bar{W}))=Z^{0} \bar{W}^{0}-Z^{1} \bar{W}^{1}-\cdots-Z^{d} \bar{W}^{d} .
\end{gathered}
$$


Then

$$
\langle g, A\rangle=\frac{\left\langle G,\left(\left(Z_{2}, \bar{W}\right)\right) Z_{1}-\left(\left(Z_{1}, \bar{W}\right)\right) Z_{2}\right\rangle}{\left(\left(Z_{1}, \bar{W}\right)\right)^{\nu+1}\left(\left(Z_{2}, \bar{W}\right)\right)^{\nu+1}} .
$$

If we view $G$ as a (horisontal) form homogeneous of degree $-\mu$ (recall that $\mu=2 \nu+1=2(\alpha+1+d)+1)$, the $\mathrm{SU}(1, d)$-invariant character of our formula is manifest.

It is now easy to make the transition to general $s>1$.

Let $G=G(W)(\cdot)$ denote an $s$-linear form homogeneous of degree $-\mu$ in $W$ (where now $\mu=2 \nu+s=2(\alpha+1+d)+s$ ) and horisontal in the sense that

$$
G(W)(\ldots, W, \ldots)=0 .
$$

Then we may consider the kernel

$$
\mathscr{A}\left(z_{1}, z_{2}\right)=\int_{\mathbf{B}} \frac{\left\langle G,\left(\left(\left(Z_{2}, \bar{W}\right)\right) Z_{1}-\left(\left(Z_{1}, \bar{W}\right)\right) Z_{2}\right)^{\otimes s}\right\rangle}{\left(\left(Z_{1}, \bar{W}\right)\right)^{\nu+s}\left(\left(Z_{2}, \bar{W}\right)\right)^{\nu+s}}\|W\|^{2 \mu} d I(w),
$$

where thus $\nu=\alpha+1+d, \mu=2 \nu+1$, and $I$ is the $\mathrm{S}(1, d)$-invariant measure on $\mathbf{B}$. Note that the integrand is homogenous of degree 0 and so, projecting into $\mathbf{P}^{d}$, can be regarded as a function on $\mathbf{B}$. Therefore the integral makes sense.

\section{Generalizing the transvectant}

Again we start with $s=1$.

Let us introduce bilinear operators $J^{i}(i=1, \ldots, d)$, setting

$$
J^{i}\left(f_{1}, f_{2}\right)(w)=\int_{\mathbf{B} \times \mathbf{B}} \overline{A^{i}\left(z_{1}, z_{2}, w\right)} f_{1}\left(z_{1}\right) f_{2}\left(z_{2}\right) d M_{\alpha}\left(z_{1}\right) d M_{\alpha}\left(z_{2}\right)
$$

so that the bilinear form $H=H_{g}$ can be written

$$
H\left(f_{1}, f_{2}\right)=\int_{\mathbf{B}} \sum_{i=1}^{d} J^{i}\left(f_{1}, f_{2}\right)(w) \overline{g_{i}(w)} d M_{\beta}(w),
$$

where as before $\beta+1+d=2(\alpha+1+d)$ or $\mu=2 \nu+1$.

Take first $w=0$. Integrating by parts, as in the final remark in Section 1 , we find

$$
J^{i}\left(f_{1}, f_{2}\right)(0)=\sum_{i=1}^{d} \frac{\partial f_{1}(0)}{\partial w^{i}} f_{2}(0)-\frac{\partial f_{2}(0)}{\partial w^{i}} f_{1}(0)
$$

On the other hand, it is easy to see that the operators $J^{i}$ enjoy the following 
transformation property

$$
\sum_{k=1}^{d} J^{k}\left(\tilde{f}_{1}, \tilde{f}_{2}\right)(z) \frac{\overline{\partial \phi^{i}}}{\partial z^{k}}(E(z))^{\mu} \bar{E}(z)=J^{i}\left(f_{1}, f_{2}\right)(\phi z),
$$

where $\tilde{f}_{1}(z)=f_{1}(\phi z) E(z)^{-\nu}, \tilde{f}_{2}(z)=f_{2}(\phi z) E(z)^{-\nu}$.

Indeed, we find

$$
\begin{aligned}
& \sum_{i=1}^{d} J^{k}\left(\tilde{f}_{1}, \tilde{f}_{2}\right)(w) \frac{\overline{\partial \phi^{l}}}{\partial z^{k}}(E(w))^{\mu} \overline{E(w)} \\
& =\sum_{i=1}^{d} \int_{\mathbf{B} \times \mathbf{B}} \overline{A^{k}\left(z_{1}, z_{2}, w\right)} f_{1}\left(\phi z_{1}\right)\left(E\left(z_{1}\right)\right)^{-\nu} f_{2}\left(\phi z_{1}\right)\left(E\left(z_{1}\right)\right)^{-\nu} d M_{\alpha}\left(z_{1}\right) d M_{\alpha}\left(z_{2}\right) \\
& \quad \times \frac{\overline{\partial \phi^{i}}}{\partial w^{k}}(E(w))^{\mu} \overline{E(w)} \\
& \left.\left.=\int_{\mathbf{B} \times \mathbf{B}} \overline{A^{i}\left(\phi z_{1}, \phi z_{2}, \phi w\right)} \overline{\left(E\left(z_{1}\right)\right.}\right)^{-\nu}\left(\overline{E\left(z_{2}\right)}\right)^{-\nu}(E(w))^{-\nu} \overline{(E(w)}\right)^{-1} f_{1}\left(\phi z_{1}\right) f_{2}\left(\phi z_{1}\right) \\
& =\int_{\mathbf{B} \times \mathbf{B}} \overline{A^{i}\left(\phi z_{1}, \phi z_{2}, \phi w\right)} f_{1}\left(\phi z_{1}\right) f_{2}\left(\phi z_{1}\right) d M_{\alpha}\left(z_{1}\right) d M_{\alpha}\left(z_{2}\right) \\
& =J^{\imath}\left(f_{1}, f_{2}\right)(\phi w),
\end{aligned}
$$

where we used that $d M_{\alpha}(\phi z)=|E(z)|^{-2 \nu} d M_{\alpha}(z)$. This establishes (2).

Now take $z=0$ in (2) and, as in Section 2, let $\phi$ be the symmetry interchanging 0 and $w$, where we first assume $w=(t, 0, \ldots, 0), t>0$. This gives

$$
J^{i}\left(f_{1}, f_{2}\right)(w)=\sum_{k=1}^{d} \frac{\overline{\partial \phi^{i}(0)}}{\partial z^{k}} J^{k}\left(\tilde{f}_{1}, \tilde{f}_{2}\right)(0)(E(0))^{\mu} \overline{E(0)} .
$$

Also by (1)

$$
\begin{aligned}
& J^{k}\left(\tilde{f}_{1}, \tilde{f}_{2}\right)(0) \\
= & \left.\left\{\frac{\partial\left(\left(f_{1}^{\circ} \phi\right) E^{-\nu}\right)}{\partial z^{k}}\left(f_{2}^{\circ} \phi\right) E^{-\nu}-\frac{\partial\left(\left(f_{2}^{\circ} \phi\right) E^{-\nu}\right)}{\partial z^{k}}\left(f_{1}^{\circ} \phi\right) E^{-\nu}\right\}\right|_{z=0} \\
= & \sum_{j=1}^{d}\left(\frac{\partial f_{1}(w)}{\partial w^{j}} \frac{\partial \phi^{j}(0)}{\partial z^{k}} f_{2}(w)-\frac{\partial f_{2}(w)}{\partial w^{j}} \frac{\partial \phi^{j}(0)}{\partial z^{k}} f_{1}(w)\right)(E(0))^{-2 \nu} .
\end{aligned}
$$

Thus

$$
J^{i}\left(f_{1}, f_{2}\right)(w)=\sum_{j=1}^{d} \sum_{k=1}^{d} \frac{\overline{\partial \phi^{i}(0)}}{\partial z^{k}} \frac{\partial \phi^{j}(0)}{\partial z^{k}}\left(\frac{\partial f_{1}(w)}{\partial w^{j}} f_{2}(w)-\frac{\partial f_{2}(w)}{\partial w^{j}} f_{1}(w)\right) .
$$


By Section 2

$$
\sum_{k=1}^{d} \frac{\overline{\partial \phi^{i}(0)}}{\partial z^{k}} \frac{\partial \phi^{j}(0)}{\partial z^{k}}= \begin{cases}\left(1-t^{2}\right)^{2} & i=j=1 \\ 1-t^{2} & i=j>1 \\ 0 & i \neq j\end{cases}
$$

So $($ as $w=(t, 0, \ldots, 0))$

$$
\left\{\begin{array}{l}
J^{1}\left(f_{1}, f_{2}\right)=\left(1-t^{2}\right)\left(\frac{\partial f_{1}(w)}{\partial w^{1}} f_{2}(w)-\frac{\partial f_{2}(w)}{\partial w^{1}} f_{1}(w)\right) \\
J^{k}\left(f_{1}, f_{2}\right)=\frac{\partial f_{1}(w)}{\partial w^{k}} f_{2}(w)-\frac{\partial f_{2}(w)}{\partial w^{k}} f_{1}(w) \quad(k>1) .
\end{array}\right.
$$

Passing to general $w$ this finally gives

$$
J^{i}=\left(\nabla^{i} f_{1}\right) f_{2}-f_{1}\left(\nabla^{i} f_{2}\right)
$$

where (cf. [R])

$$
\nabla^{i}=\frac{\partial}{\partial z^{i}}-\bar{z}^{i} R, \quad R=\sum_{i=1}^{d} z^{i} \frac{\partial}{\partial z^{i}} .
$$

Time for the Janson remark (see again [P])! We put $g_{0}=-\sum_{i=1}^{d} z_{i} g_{i}$ and consider the form

$$
\begin{aligned}
G & =\left(Z^{0}\right)^{-\mu} \sum_{i=0}^{d} g_{i}\left(\frac{Z^{1}}{Z^{0}}, \ldots \frac{Z^{d}}{Z^{0}}\right) d Z^{i} \\
& =\left(Z^{0}\right)^{-2 \nu} \sum_{i=1}^{d} g_{i}\left(\frac{Z^{1}}{Z^{0}}, \ldots, \frac{Z^{d}}{Z^{0}}\right) d\left(\frac{Z^{i}}{Z^{0}}\right)
\end{aligned}
$$

We further set

$$
F_{1}=\left(Z^{0}\right)^{-\nu} f_{1}\left(\frac{Z^{1}}{Z^{0}}, \ldots, \frac{Z^{d}}{Z^{0}}\right), \quad F_{2}=\left(Z^{0}\right)^{-\nu} f_{2}\left(\frac{Z^{1}}{Z^{0}}, \ldots, \frac{Z^{d}}{Z^{0}}\right) .
$$

Then

$$
\begin{aligned}
\sum_{i=1}^{d} J_{i} \bar{g}_{i} & =\sum_{i=0}^{d} \pm\left(\frac{\partial F_{1}}{\partial Z^{i}} F_{2}-\frac{\partial F_{2}}{\partial Z^{i}} F_{1}\right) \bar{g}^{t} \\
& =\left(\left(d F_{1} \cdot F_{2}-F_{1} d F_{2}, G\right)\right)
\end{aligned}
$$

so (1) can be written as 


$$
H\left(f_{1}, f_{2}\right)=\int_{\mathbf{B}}\left(\left(d F_{1} \cdot F_{2}-F_{1} d F_{2}, G\right)\right)\|Z\|^{2 \mu} d I(Z)
$$

As in Section 2, we observe that the integrand in this formula is homogenous of degree 0 .

It is now easy to write down the generalization to $s>1$. For instance, if $s=2$ we take

$\left(\left(d^{2} F \odot F_{2}-2 \frac{\alpha+1+d+1}{\alpha+1+d} d F_{1} \odot d F_{2}+F_{1} \odot d^{2} F_{2}, G\right)\right)$ etc.

\section{Big Hankel operators}

So far we have only been concerned with Hankel forms, that is "small" Hankel operators. Now we turn to "big" Hankel operators. In other words, we are concerned with operators $T$ of the type

$$
T f\left(z_{1}\right)=\int_{\mathbf{B}} \overline{\mathscr{A}\left(z_{1}, z_{2}\right)} K\left(z_{1}, z_{2}\right) f\left(z_{2}\right) d M_{\alpha}\left(z_{2}\right),
$$

mapping $A^{\alpha, 2}(\mathbf{B})$ into its orthogonal complement $A^{\alpha, 2}(\mathbf{B})^{\perp}$, where $K$ is the reproducing kernel in $A^{\alpha, 2}(\mathbf{B})$ and $\mathscr{A}$ a kernel which transforms as

$$
\mathscr{A}\left(z_{1}, z_{2}\right) \mapsto \mathscr{A}\left(\phi z_{1}, \phi z_{2}\right) .
$$

This leads us to take

$$
\mathscr{A}\left(z_{1}, z_{2}\right)=\int_{\mathbf{B}} \frac{\left\langle G,\left(\left(\left(Z_{2}, \bar{W}\right)\right) Z_{1}-\left(\left(Z_{1}, \bar{W}\right)\right) Z_{2}\right)^{\otimes s}\right\rangle}{\left(\left(Z_{1}, \bar{W}\right)\right)^{s}\left(\left(Z_{2}, \bar{W}\right)\right)^{s}}\|W\|^{2 s} d I(w) .
$$

\section{Boundedness and trace ideal membership}

So far all considerations have been purely formal. It is non time to ask the usual questions about boundedness (in the space $A^{\alpha, 2}(\mathbf{B})$ ) and membership in trace ideal (Schatten-von Neumann) classes. However, we postpone this to a subsequent publication.

Note added May 1993. The results of this paper have been generalized to the case of general bounded symmetric domains in a forthcoming paper in the Rocky Mt. J. Math. 


\section{REFERENCES}

[JP] S. Janson-J. Peetre, A new generalization of Hankel operators (the case of higher weights), Math. Nachr., 132 (1987), 313-328.

[P] J. Peetre, Reproducing formulae for holomorphic tensor fields, Boll. U. M. I., (7) 2-B (1988), 343-359.

[R] W. Rudin, Function theory on the unit ball of $\mathbf{C}^{n}$, Springer, New YorkHeidelberg-Berlin, 1980.

Matematiska institutionen

Box 6701

S-113 85 Stockholm

Sweden

Present address:

Matematiska institutionen

Box 118

S-22100 Lund

Sweden 